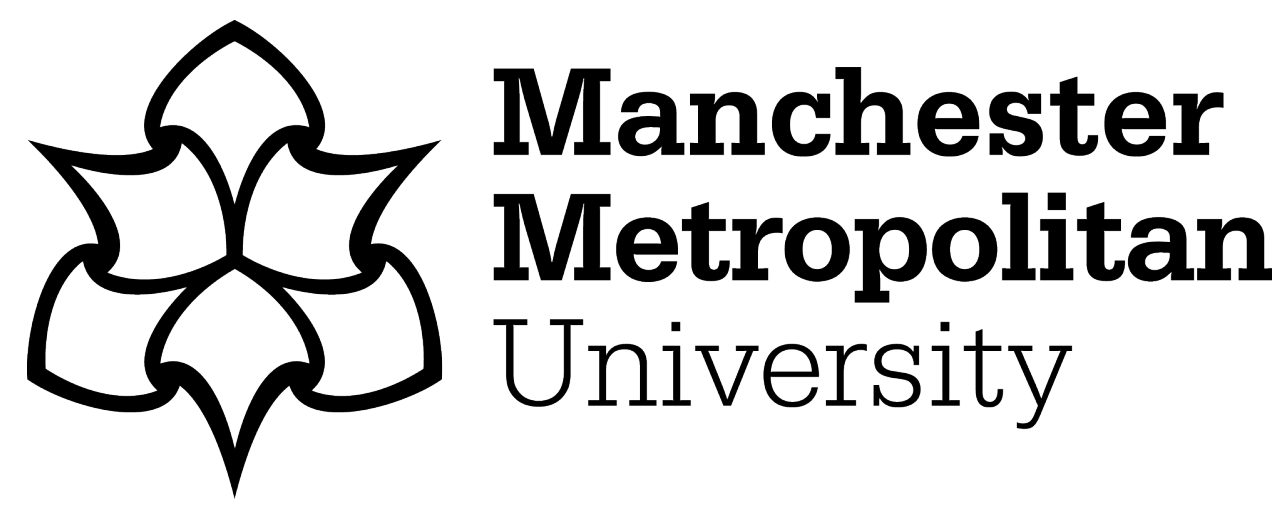

Feng, J, Wang, N, Wang, Y, Tang, X and Yuan, J (2020) Haemodynamic mechanism of formation and distribution of coronary atherosclerosis: A lesion-specific model. Proceedings of the Institution of Mechanical Engineers, Part H: Journal of Engineering in Medicine, 234 (11). pp. 1187-1196. ISSN 0954-4119

Downloaded from: https://e-space.mmu.ac.uk/626907/

Version: Accepted Version

Publisher: Sage

DOI: https://doi.org/10.1177/0954411920947972

Usage rights: Creative Commons: Attribution-Noncommercial-No Derivative Works 4.0

Please cite the published version 


\title{
Haemodynamic mechanism of formation and distribution of coronary atherosclerosis: A lesion-specific model
}

\author{
Jiling Feng ${ }^{\top}$, , Nannan Wang ${ }^{2}$, Yiliang Wang ${ }^{2}$, \\ Xiaoxian Tang ${ }^{3}$ and Jie Yuan ${ }^{3}$
}

\begin{abstract}
Coronary arterial disease, as the most devastated cardiovascular disease, is caused by the atherosclerosis in the coronary arteries, which blocks the blood flow to the heart, resulting in the deficient supply of oxygen and nutrition to the heart, and eventually leading to heart failure. To date, haemodynamic mechanisms for atherosclerosis development are not fully understood although it is believed that the haemodynamic disturbance at the region of the arterial bifurcation, particular, bifurcation angle, plays an important role in the atherosclerosis development. In this study, two types of computational fluid dynamics models, lesion-specific and idealized models, combined with the computer tomography imaging techniques, are used to explore the mechanism of formation and distribution of the atherosclerosis around the bifurcation of left coronary artery and its association with the bifurcation angle. The lesion-specific model is used to characterize the effect of personalized features on the haemodynamic performance. While the idealized model is focusing on the effect of single factor, bifurcation angle, on the haemodynamic performance. The simulated results from both types of the models, combined with the clinical observation, revealed that the three key areas around the bifurcations are prone to formation of the atherosclerosis. Unlike the idealized models, lesion-specific modelling results did not show the significant correlation between the wall shear stress and bifurcation angle, although the mean value of the wall shear stress in smaller bifurcation angles (less than $90^{\circ}$ ) is higher than that with larger bifurcation angles (greater than $90^{\circ}$ ). In conclusion, lesion-specific computational fluid dynamics modelling is an efficient and convenient way to predict the haemodynamic performance around the bifurcation region, allowing the comprehensive information for the clinicians to predict the atherosclerosis development. The idealized models, which only focus on single parameter, may not provide the sufficient and reliable information for the clinical application. A novel multi-parameters modelling technique, therefore, is suggested to be developed in future, allowing the effects of many parameters on the haemodynamic performance to be evaluated.[AQ: I]
\end{abstract}

\section{Keywords}

Left coronary artery, bifurcation angles, lesion-specific model, computational fluid dynamics modelling, atherosclerosis

Date received: 4 November 2019; accepted: I July 2020

\section{Introduction}

Cardiovascular disease (CVD) is the leading cause of death and disability in China and around the world. ${ }^{1,2}$ Over the last 30 years, incidence of the CVD has increased significantly due to the change of lifestyles, urbanization and the accelerated ageing process. It is estimated, currently, that there are approximately 0.29 billion CVD patients in China and most of the victims have ischaemic heart disease caused by the atherosclerosis in the coronary arteries. Over the past years, the fatality rate caused by CVD increases approximately $5.05 \%$ per year and this pattern remains an upward trend in the next decade. Up until now, the fatality rate caused by CVD in China and around the world accounts for more than $40 \%$ of all deaths, higher than fatality caused by cancers or any other diseases. ${ }^{2}$

\footnotetext{
'Department of Engineering, Faculty of Science and Engineering, Manchester Metropolitan University, Manchester, UK

${ }^{2}$ Department of Mechanical Design, College of Mechanical Engineering, Taiyuan University of Technology, Taiyuan, P.R. China

${ }^{3}$ Radiology Department, Shanxi Provincial People's Hospital, Taiyuan, P.R. China
}

\section{Corresponding author:}

jiling Feng, Department of Engineering, Faculty of Science and Engineering, Manchester Metropolitan University, Manchester MI 5GD, UK.

Email: J.feng@mmu.ac.uk 
Coronary arterial disease (CAD) is the most clinical complication of CVD. The atherosclerosis developed in the coronary artery is major cause of CAD, which reduces the lumen of coronary arteries and blocks the blood flow to the heart, resulting in deficient supply of the oxygen and nutrition to the heart, eventually leading to the heart failure. Over the past few decades, the mechanism of CAD has been extensively investigated. It has been widely accepted that the haemodynamic disturbance at the region of the arterial bifurcation plays an important role in the atherosclerosis development in the coronary artery. Particularly, the bifurcation angle (BA), which is between the daughter vessels after branching, has been suggested as a risk predictor in clinic of cardiovascular diseases. ${ }^{3}$ Especially locations with low and oscillated wall shear stress (WSS) near the artery bifurcation are susceptible to the atherosclerosis development. ${ }^{4}$

The anatomic analysis of the coronary artery has identified that the left coronary artery (LCA) has a higher risk of the atherosclerosis due to the high disturbance and separation of the blood flow at this area. Based on the clinical observations from the LCA computer tomography (CT) images, Gaston demonstrated that the plaques were prone to grow at local bifurcation and near the opposite wall of branches. ${ }^{6}$ Tang et al. found from the CT images that BA in the LCA has a strong correlation with the distribution of the atherosclerosis. They noticed that the atherosclerosis occurred at branch of the left anterior descending (LAD) artery more frequently than the other sites. ${ }^{7}$ Juan et al. ${ }^{8}$ also observed that patients with significant stenosis in the LCA have the larger BA.

The mechanisms of the CAD have also been explored using the numerical modelling techniques. Over the past decades, significant efforts have been made to distinguish the haemodynamic performance and the coronary arteries disease using the advanced computational fluid dynamics (CFD) techniques. Dong et al. ${ }^{9}$ developed a fluid-structure interaction (FSI) model to investigate the correlation of BA with the atherosclerosis distribution. They found that BA is positive associated with the circumferential stress of the vessel wall. ${ }^{9}$ The numerical study from Antoniadis ${ }^{10}$ observed that local WSS has a significant effect on the formation of the atherosclerosis. He concluded that CFD modelling, combined with the advanced imaging technique, is an efficient technique to diagnose the atherosclerosis. Although it is accepted that BA has an effect on the formation of the atherosclerosis, there are some controversial arguments about the effects of BAs on the atherosclerosis formation. For example, Beier had argued that the BA has less effect on the haemodynamic performance, by comparing effects of other two parameters such as the size of the cross-section areas and the degree of bending. ${ }^{11}$

Up to date, the underpinning mechanism of the development of atherosclerosis has not been fully understood despite significant efforts have being made. ${ }^{12-15}$ This study aims to employ an emerging technique, lesion-specific CFD modelling, combining with the advanced CT imaging techniques, to explore the mechanism of formation and distribution of the atherosclerosis and its association with the BA. In this study, a total of 24 multisliced CT images of the LCA were selected by clinical collaborators from a local hospital database in China. Two types of three-dimensional (3D) CFD models, the idealized and the lesion-specific models, were constructed to reveal the haemodynamic performance of the LCA, particularly around the range of the bifurcation. The haemodynamic parameters, obtained through the idealized and patient-specific CFD models, were compared with the atherosclerosis distributions within the vessel observed from the CT images.

\section{Materials and methods}

Twenty-four original Phillips Digital Imaging and Communications in Medicine (DICOM) CT images of the coronary artery were selected from database of symptomatic patients in local hospital in China. The details of the study were informed to the patients involved in this study and the consent form was signed. The ethical form was approved by ethical committee in the hospital before the data collection. The multisliced CT images in DICOM format were imported into the commercial software MIMICS for the reconstruction of the three branches of the LCA model. The interested segments - including left main coronary (LMC), left LAD and left circumflex coronary (LCx) artery - were extracted using semi-automatic technique. In this study, two types of the CFD models, idealized and lesion-specified models, were developed to investigate the effect of BA on the haemodynamic performance in the LCA. The lesion-specific CFD models were developed from nine selected LCA images with a wide range of BAs, which enables the correlation of flow performance with BAs to be assessed. The idealized models were constructed based on a typical example of CT image, which allows for the geometries of the all models kept identical with the exception of the BAs varied in a wide range; therefore, the univariate effect of BA on the flow performance can be evaluated.

\section{Lesion-specific model}

The lesion-specific model for the LCA, including three branches, was reconstructed using commercial software based on CT image (Figure 1). As is emphasized above, this study focused on the effect of BA angles on the haemodynamic performance; therefore, we selected nine cases of LCA out of 24 images with a wide range of angles from $45.8^{\circ}$ to $121.2^{\circ}$ to construct CFD models (Figure 2). In order to minimize the effect of the other parameters on the flow performance, the minimum variation of the other parameters - such as curvature of the each branch and tortuosity - was considered 


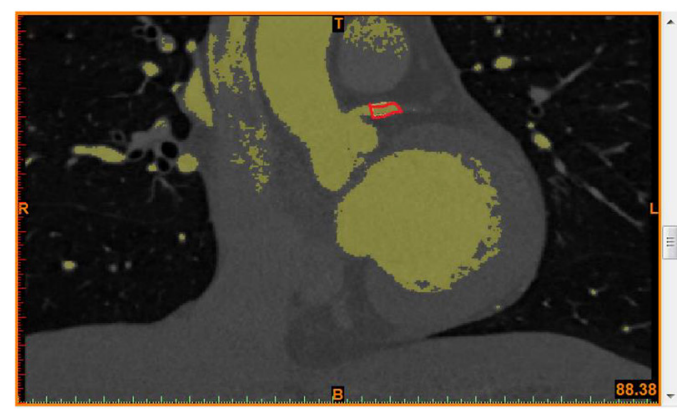

(a)

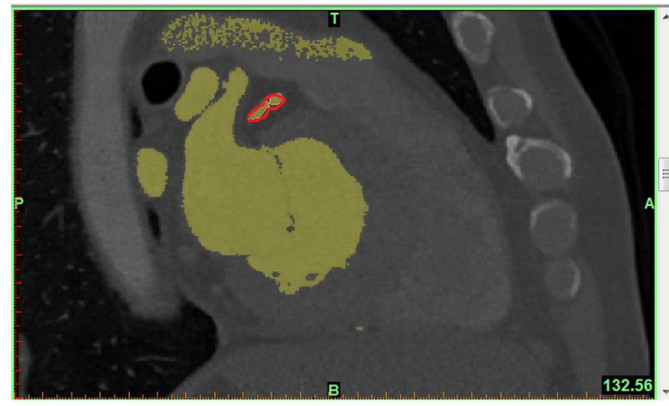

(c)

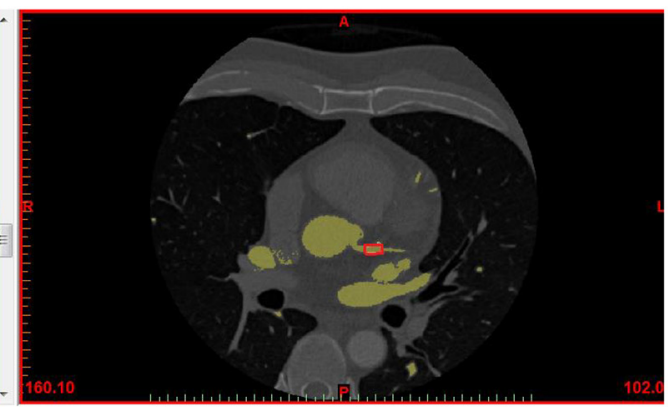

(b)

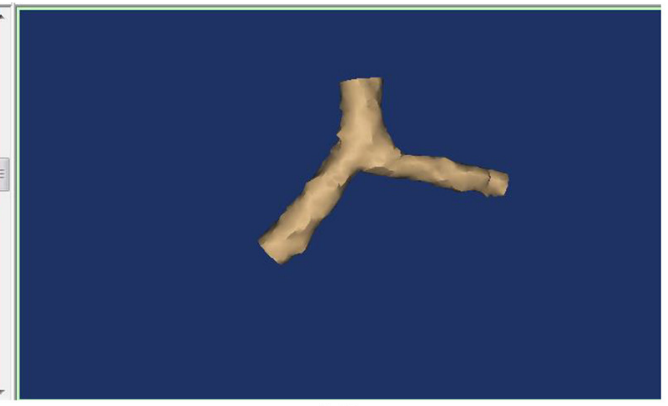

(d)

Figure I. Extraction of left coronary model using MIMICS. (a)-(c) are MSCT slices with three perspectives with the left coronary artery (marked with red cycle) and (d) is the restructured 3D model of left coronary artery (LCA).

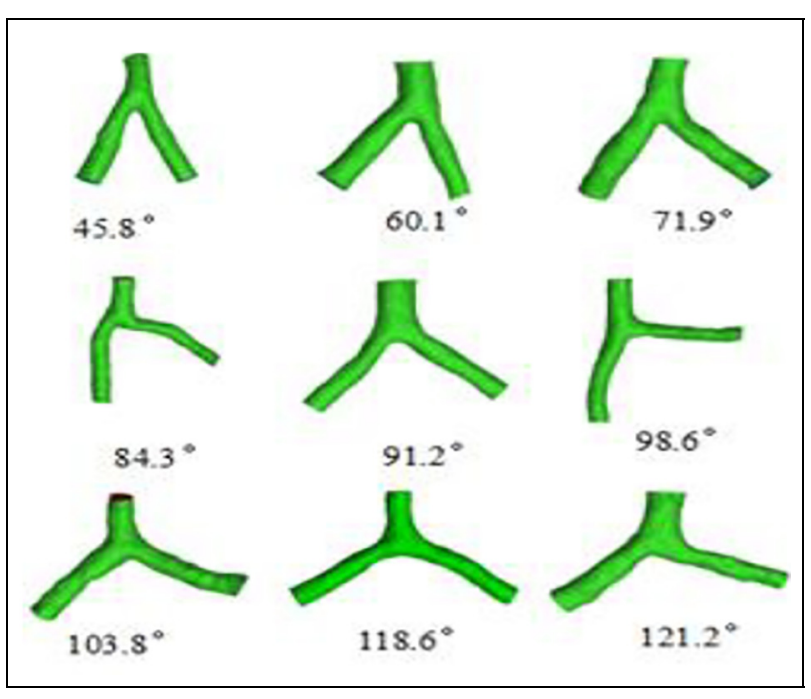

Figure 2. Nine lesion-specific left coronary models with the varied bifurcation angles.

as the criteria for the selection of the images. Within 24 images, the minimum degree of lesion was also considered for the selection of the images. The 3D geometrical LCA model - consisting with LMC, LAD coronary and $\mathrm{LCX}$ - was reconstructed, and then transferred to the commercial software ANSYS for CFD simulation.

\section{The idealized model}

In order to investigate the effects of BA to the formation of atherosclerosis, a total of five idealized models with the different BA were developed to investigate the haemodynamic performance in the LCA. In the previous study, the cut-off value of $80^{\circ} \mathrm{BA}$ was suggested as the threshold for the atherosclerosis development. ${ }^{8}$ In this study, medium value of BA is $91.2^{\circ}$; therefore, the image with this angle was selected as a reference image to construct five idealized models. Five idealized LCA models, with angles between $\mathrm{LAD}$ and $\mathrm{LCx}$ ranging from $60^{\circ}$ to $120^{\circ}$ at interval of $15^{\circ}$, were developed. The models characterized with axis-symmetrical and elliptical cross-sectional shape (Figure 3(a) and (b)). The main branch and two daughter branches share the same plane where LAD and LCx are symmetrical about the central line of LCM.[AQ: 2] The angles between LAD and $\mathrm{LCX}$ are controlled as the only variable quantity in order to eliminate effects from other parameters, such as length, cross-sectional area and curvature.

\section{CFD configuration and physiological parameters}

CFD simulation was carried out using the ANSYS FLUENT (ANSYS, Inc., Canonsburg, PA, USA) on a Microsoft Windows 7 64-bit machine, 256 MB RAM with a dual core $2.0 \mathrm{GHz}$ CPU. The commercialized software ICEM CFD was used for meshing, where refined mesh was conducted through the geometric inspection and topology correction. Quadratic tetrahedral finite elements were generated with the average numbers of elements approximately 145,935 and nodes 25,581 . The refined meshes were particularly used around the areas near the arterial wall by divided 


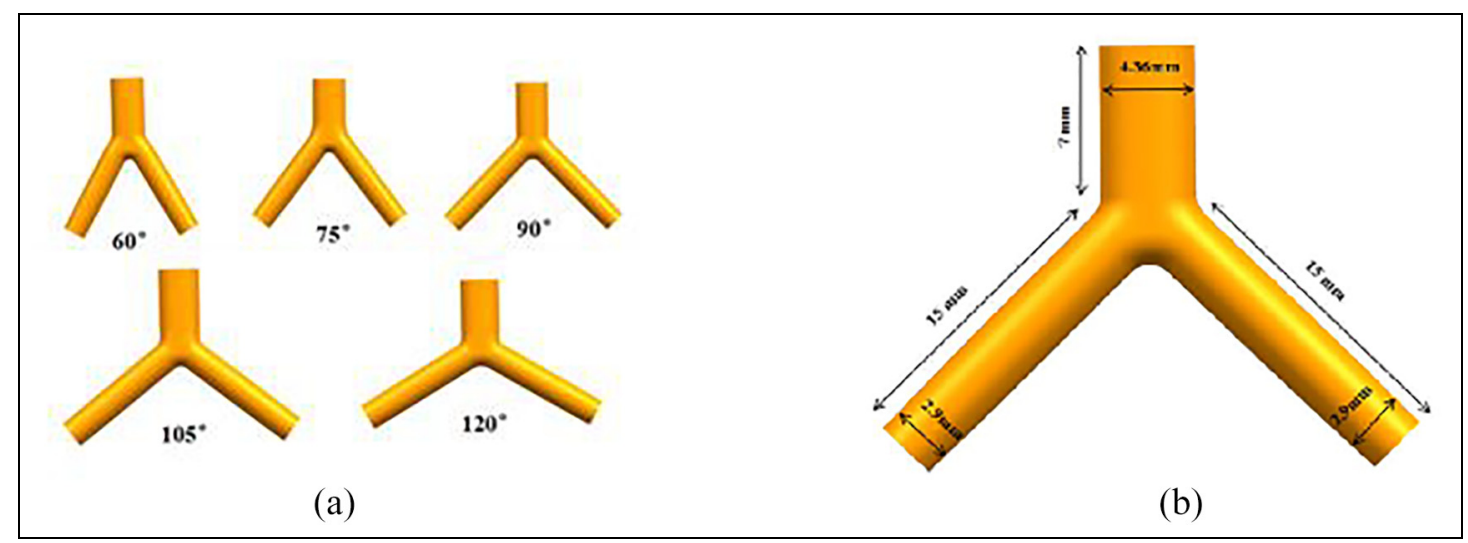

Figure 3. (a) Left coronary ideal model with different bifurcation angles and (b) a schematic diagram of the structure and size of the idealized model.

boundary layer into four layers to ensure the accuracy of the results of the flow performance, particularly for the WSS, a key factor for the atherosclerosis development. The Navier-Stokes equations were used to simulate the transient pulsatile blood flow problem within two cardiac cycles. The pulsatile aortic pressure and flow rate profiles at $\mathrm{LCA}^{16}$ were used to apply the boundary conditions to the inlet and outlet, respectively. The pulsatile pressure and flow rate profile were reconstructed using the Fourier series. The user-defined function (UDF) was edited using $\mathrm{C}$ language, representing the pulsatile pressure profile and LCA flow rate profile are applied the boundary conditions at the inlet and outlet of models. Given that lesion-specific and idealized CFD models developed with minimum variation of the parameters other than BA, 50/50 of pulsatile coronary flow profile was applied at the outlet of $\mathrm{LCx}$ and LAD, respectively. In this study, non-slip boundary conditions between the artery vessel wall and the blood flow domain are assumed. The vessel wall is assumed as rigid with no permeability, while the blood flow is considered as the Newtonian and incompressible flow under the normal physiological conditions. At the normal body temperature of $37^{\circ} \mathrm{C}$, the density and viscosity of blood flow, $\rho=1055 \mathrm{~kg} / \mathrm{m}^{3}$ and $\mu=0.0035$ Pas, are used for analysis. The blood flow in LCA can be considered as the laminar flow due to the low Reynolds number.

\section{Results}

\section{The lesion-specific models}

Velocity and WSS distribution. A total of nine lesionspecific models with a wider range of BAs were developed based on its unique patient-specific CT images. The velocity and WSS profiles for all models are presented in Figure 4(a) and (b). We expected that the strong association exists between BAs and flow patterns, however, which was not observed in this study. Indeed, some visible differences can be identified if these nine lesion-specific models are classified into two groups - the group with smaller angles $\left(45.8^{\circ}, 60.1^{\circ}\right.$, $\left.71.9^{\circ}, 84.3^{\circ}\right)$ and the group with larger angles $\left(91.2^{\circ}\right.$, $\left.98.6^{\circ}, 103.8^{\circ}, 118.6^{\circ}, 121.2^{\circ}\right)$. It was observed that the overall velocities in the group with larger angles are lower than those in the groups with the smaller angle. The common feature observed in all models is that the areas with higher velocity tend to divert into the inner vessel walls in both daughter branches. In order to observe the flow pattern in radial direction, the crosssectional views at three locations - A-A, B-B and C-C - were generated at the main coronary artery and two branches, respectively (Figure 4(c)). The cross-sectional view for the pressure and the velocity in both sections (A-A and B-B) indicated that the lower velocity and higher pressure can be found in the models with the larger BAs and vice versa (Figure 4(d)).

WSS at the key areas. Three typical regions around the bifurcation - the ridge of the bifurcation, the areas close to the opposite wall and the areas close to the inner wall of two branches - were selected to observe the WSS distribution (Figure 5(a)). The range of WSS in these three typical areas is presented in Figure 5(b). It is very clear that higher WSS occurs consistently in the areas close to the inner wall of the branches, which is 4-5 times more than the magnitude of the WSS in the areas near the opposite wall. In the ridge of the bifurcation, the magnitude of WSS is comparable to the value at the region around opposite wall. Particularly, in the ridge of bifurcation, the value of the WSS has the strong negative exponential correlation with the BAs (correlation coefficient, $R^{2}=0.86$ ). However, the correlation between BAs and WSS is weak in the other two areas. It is consistently shown that the mean value of WSS in the group of smaller angles $\left(\mathrm{BA}<90^{\circ}\right.$ ) is generally larger than that in the group of larger angles $\left(\mathrm{BA}>90^{\circ}\right)$ in all three areas. The ratios of the mean value of WSS between the group of the smaller angles and large angles are: $0.4 \mathrm{~Pa}$ via $0.14 \mathrm{~Pa}$ at the ridge of the bifurcation; $0.45 \mathrm{~Pa}$ via $0.31 \mathrm{~Pa}$ at the area near the 


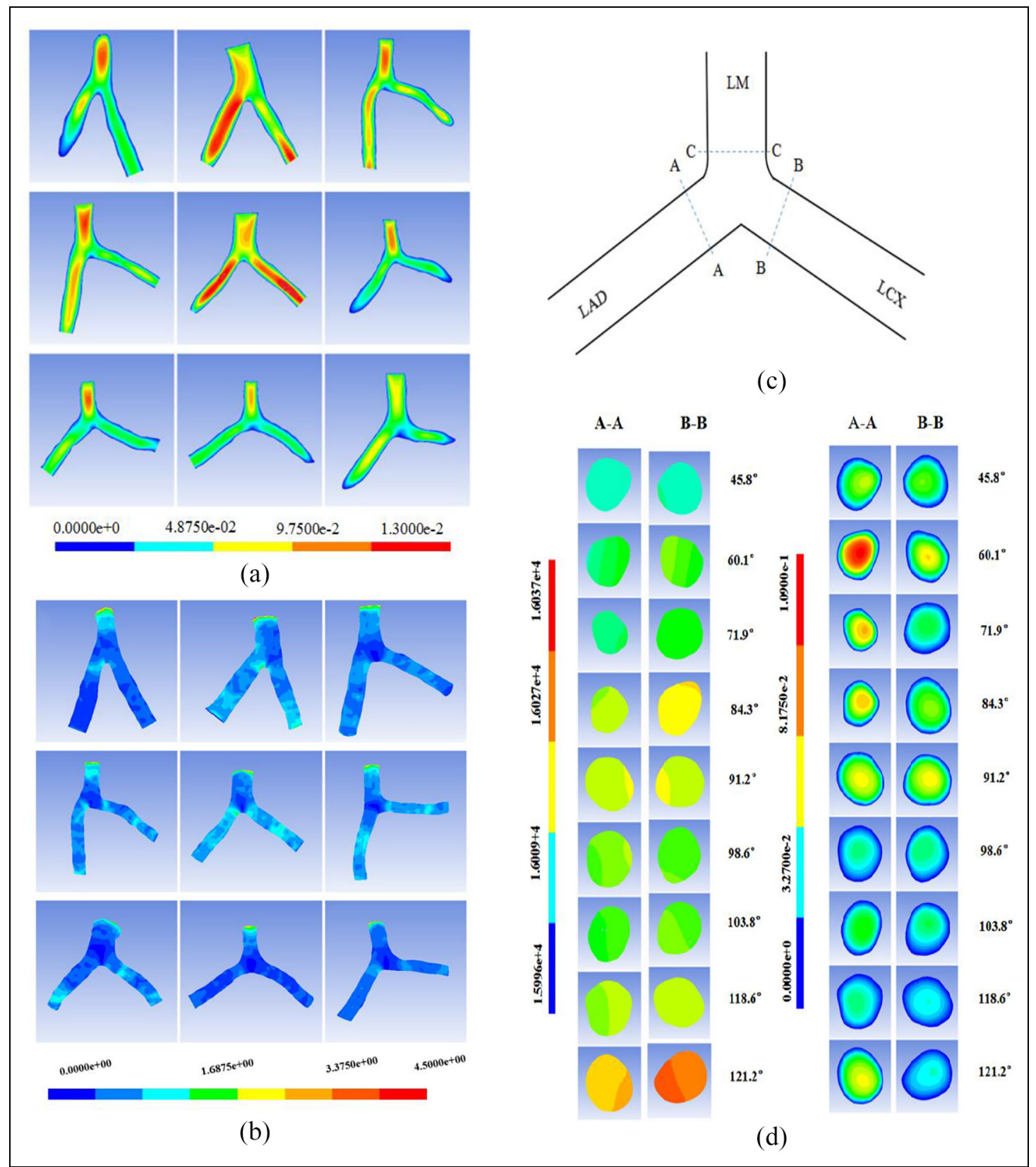

Figure 4. The contour of the velocity in $\mathrm{m} / \mathrm{s}(\mathrm{a})$ and WSS in Pa (b) for nine lesion-specific CFD models at the time point of $0.4 \mathrm{~s}$, (c) schematic diagram for locations of the three key cross-sectional area and (d) the cross-sectional view of the pressure and the velocity at $0.4 \mathrm{~s}$ for the left coronary artery at different bifurcation angles at $0.4 \mathrm{~s}$ (the pressure (left) and velocity (right)).

inner wall; $1.83 \mathrm{~Pa}$ via $1.68 \mathrm{~Pa}$ at the area close to opposite wall.

Atherosclerosis distribution in the $L A D$ and $L C x$. Clinical observations for the distribution of atherosclerosis in the branches of the LAD and LCx were analysed statistically by radiologists based on the same group of CT images. As is shown in Figure 6(a), the cross-sections (A-A in the LCx and B-B in the LAD) were participated into four quadrants (QI: the area close to inner vessel wall; QIII: the area close to the opposite vessel wall; QII: the area next to the pericardial side; QIV: the area closes to myocardium side). The clinical observation indicated that nearly $80 \%(19 / 24)$ of the atherosclerosis occurs in the QIII, where only one case with the $45.5^{\circ}$ occurs in the QI (Table 1 and Figure 6(b)). The clinical observations for the regions of atherosclerosis occurring most likely are consistent with the simulated results for the low WSS regions, indicating the interrelation of the formation of atherosclerosis and haemodynamic mechanisms.

\section{Idealized model}

Figure 7(a)-(c) shows the contour profile of the velocity, pressure and WSS in the end of the systolic period 


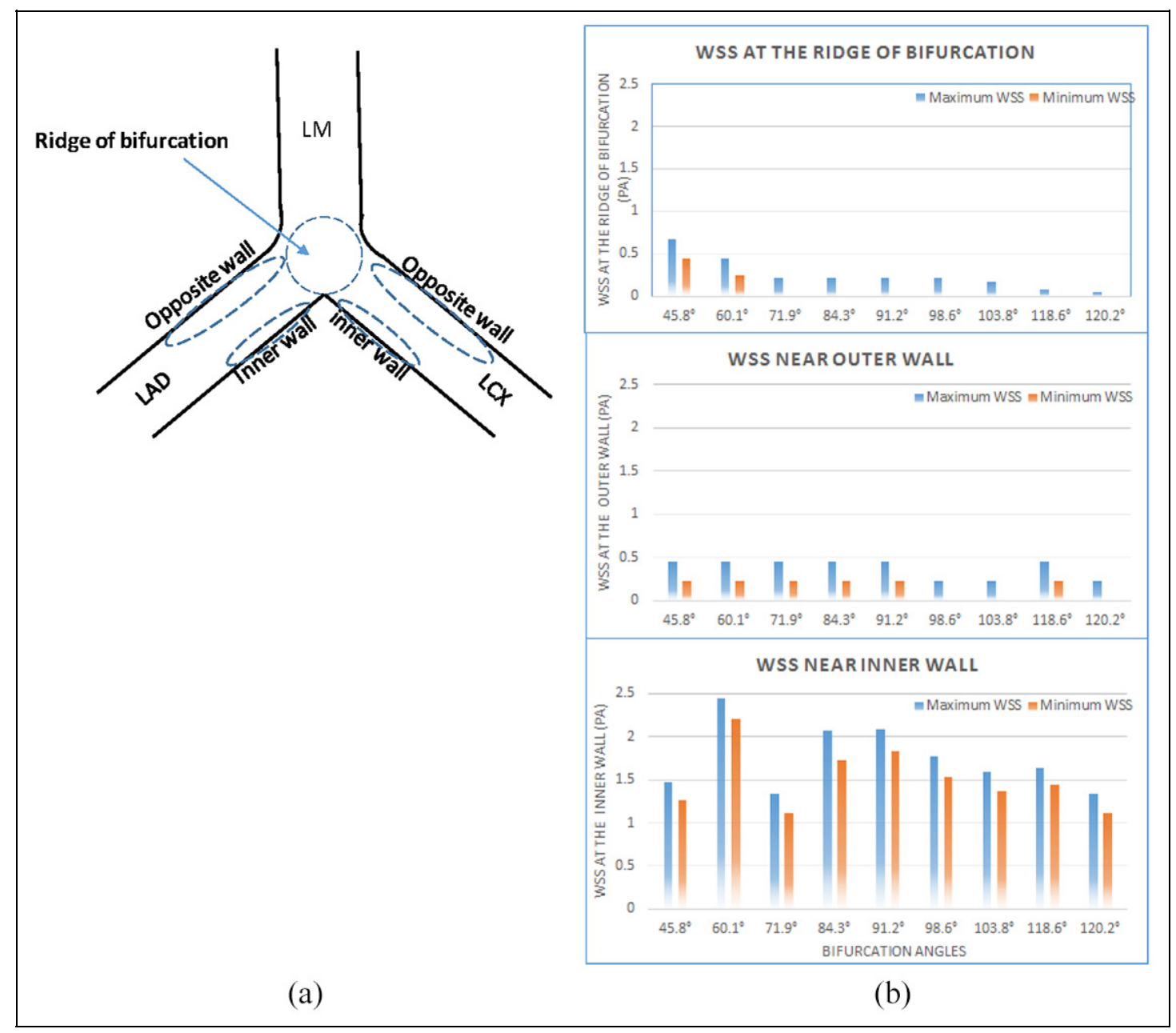

Figure 5. [AQ: 3]The WSS in three key areas (the ridge of bifurcation, area closer to the inner and opposite walls).

Table I. The clinical observation for the atherosclerosis distribution in the four-quadrant area.

\begin{tabular}{llllll}
\hline Range of BAs & Number of items & QI & QII & QIII & QIV \\
\hline$<60^{\circ}$ & 9 & 1 & 4 & 4 & 0 \\
$60^{\circ}-90^{\circ}$ & II & 0 & 0 & II & 0 \\
$90^{\circ}-120^{\circ}$ & 3 & 0 & 0 & 3 & 0 \\
$>120^{\circ}$ & I & 0 & 0 & 1 & 0
\end{tabular}

BAs: bifurcation angles.

(time $=0.4 \mathrm{~s})$, the moment when the maximum pressure and the first peak of blood flow occur. It was noticed, at the ridge of bifurcation, that the area of the low velocity (green) gradually becomes larger when the degree of angulation increases (Figure 7(a)). The similar pattern was also observed in the pressure contour, where region of the high pressure broadens when the angles increase (Figure 7(b)). This phenomenon indicates that the bifurcation with the wider angle resulted in a significant change of the flow directions in the branches of arteries, consequently, creating a greater impact force and high pressure acting on the bifurcation. The reaction force from the ridge of bifurcation causes the retrograde flow, leading to the dramatic reduction of the velocity. In Figure 7(c), one can see that the lower WSS tends to occur around the ridge of bifurcation for the models with the smaller BA (e.g. $60^{\circ}$ and $75^{\circ}$ models), while the areas of lower WSS gradually spread out towards the daughter branches as the angles increase. The variation of the velocity, pressure and WSS with the angles also can be observed by the cross-sectional views (Figure 7(d) and (e)). From the sections of A-A and $\mathrm{B}-\mathrm{B}$, it is clear that the high velocity domain (redyellow area) tends to move away from the outer vessel wall and towards the inner wall. With the increase in BAs, the high velocity area is further away from the outer wall, leading to the velocity gradient and WSS decreasing. This phenomenon also can be observed from the front view of the velocity contour, where the high velocity streamline tends to attach the inner wall of both daughter branches, resulting in the separation of the flow around the opposite wall, where the extreme low and unstable WSS occurs.

\section{Discussion}

The association of the atherosclerosis development and the BAs has been widely explored. It has been realized 


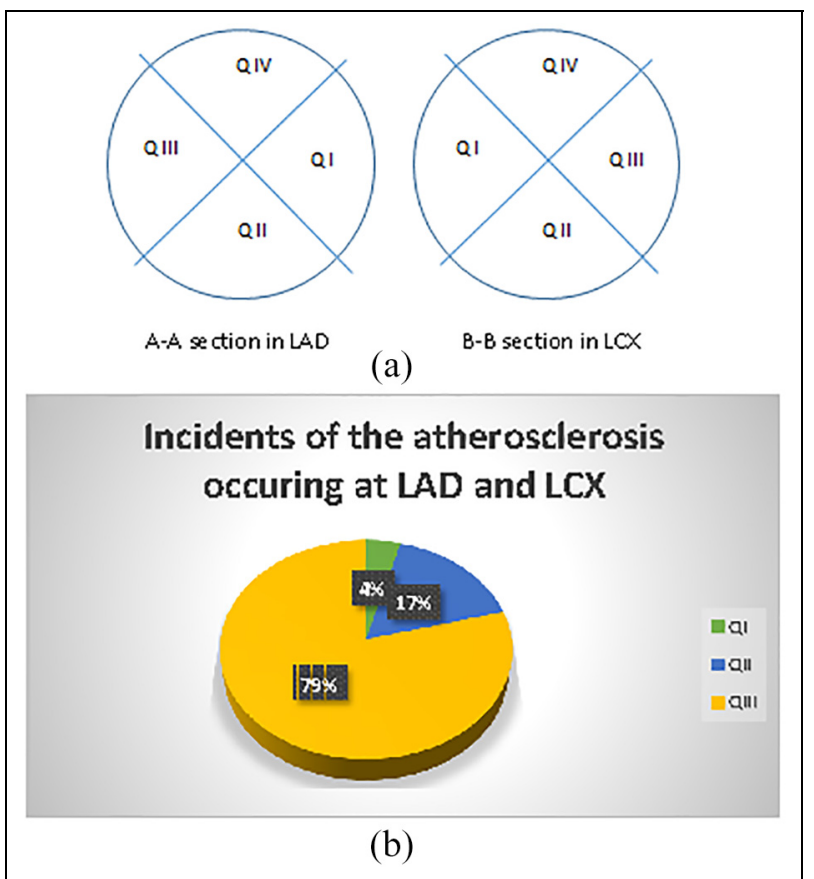

Figure 6. (a) Schematic of quadrant diagram at the crosssection in LAD and LCX and (b) pie chart for the incidents of the areas of atherosclerosis formation.

that the low WSS due to haemodynamic disturbances occur in local area plays an important role for the formation of the atherosclerosis. ${ }^{17-20}$ Chatzizisis et al. ${ }^{21}$ think that low shear stress has a continuous mechanical effect on vascular endothelial cells, which induces the activation of the endothelial cell-related factors and leads to the release of chemokines and proteins. This eventually promotes the formation of lipid and leukocyte aggregations and lesions. ${ }^{21}$ In this study, correlation of the atherosclerosis formation and distribution with the BAs and the other parameters such as the cross-sectional areas were investigated based on the lesion-specific left coronary arterial models. To eliminate the effect of the other factors, the idealized models, which possess the identical features but with varied BAs at the equal interval, were also developed to investigate the unique relationship between the atherosclerosis formation and BAs. The simulated results were compared with the clinical observation based on CT images.

\section{Prediction of lesion susceptible area}

The region around the bifurcation in the LCA is considered as the site that is more susceptible to the development of atherosclerosis. It is believed that haemodynamic disturbance due to the local geometrical discontinuity made significant contribution to it. However, the precise location of atherosclerosis and its association to the haemodynamic mechanism have not been explored in great depth. In this study, three key areas around the bifurcation regions have been identified as the locations where the atherosclerosis is mostly likely to occur. In the daughter branches (LCX and LAD), mainstream of the blood moves away from the central area to the inner side of the vessel wall. Such flow pattern leads to lower velocity domains around the outer vessel wall. It is clear that the velocity streamline is less dense in areas around the outer layer than that near the inner layer. In terms of the definition of WSS $\left(t_{w s s}=\mu(\partial U / \partial n)\right.$, where $t_{w s s}$ is the WSS, $\mu$ is the dynamic viscosity and $\partial U / \partial n$ is the gradient of velocity in the normal direction), WSS is proportional to the gradient of velocity in the normal direction.[AQ: 4] The local variation of the velocity distribution, therefore, has an immediate effect on the WSS, which led to oscillation lower WSS in the area near the opposite wall. In terms of the haemodynamic mechanism, the three typical areas - the ridge of the bifurcation, the area close to the opposite wall and the area close to the inner wall - are considered as the critical regions susceptible for atherosclerosis formation.[AQ: 5] The prediction from the simulation results is consistent with the clinical observation.

\section{The effect of BAs on the atherosclerosis formation and distribution}

The clear negative correlation between the angulations of the LCA and the lower WSS region was observed from the idealized model results. Based on theories of fluid dynamics and propagation of blood flow wave, the reflection of waves at the point of bifurcation will lead to the retrograde flow and this could reduce the flow velocities significantly. Since wider angle of bifurcation causes significant changes of flow directions and momentum, it is rational that wider angle of bifurcation will generate stronger reflection waves, thus leading to reduction of the flow velocities in the downstream. This phenomenon was clearly shown in the cross-sectional view of the velocity contour. At the ridge of bifurcation, it was found that a strong negative correlation exists between BAs and WSS. In the branches, correlation between the WSS and BAs was weak, which is in accordance with Beier et al.'s $s^{11}$ finding where they found the effect of BAs on the haemodynamic performance is less than the effect of the other parameters. The lower value of WSS was occurring in the group with smaller angles and vice versa, which could explain the finding from Juan et al. ${ }^{8}$, showing patients with significant stenosis in the LCA have the larger BA. In this study, evaluation of WSS distribution was conducted at the different time points within one cardiac cycle $(0.28,0.31,0.4,0.5$ and $0.57 \mathrm{~s}$ ). The amplitudes of the WSS changed over the time within cardiac cycle; however, the distribution of the WSS within the flow domain at five key time points is consistent. It was observed that the lowest values of WSS were occurring at 0.31 and $0.5 \mathrm{~s}$, respectively. At $0.4 \mathrm{~s}$, it reached the first peak and at $0.57 \mathrm{~s}$, the WSS achieved the maximum value within one cycle. 


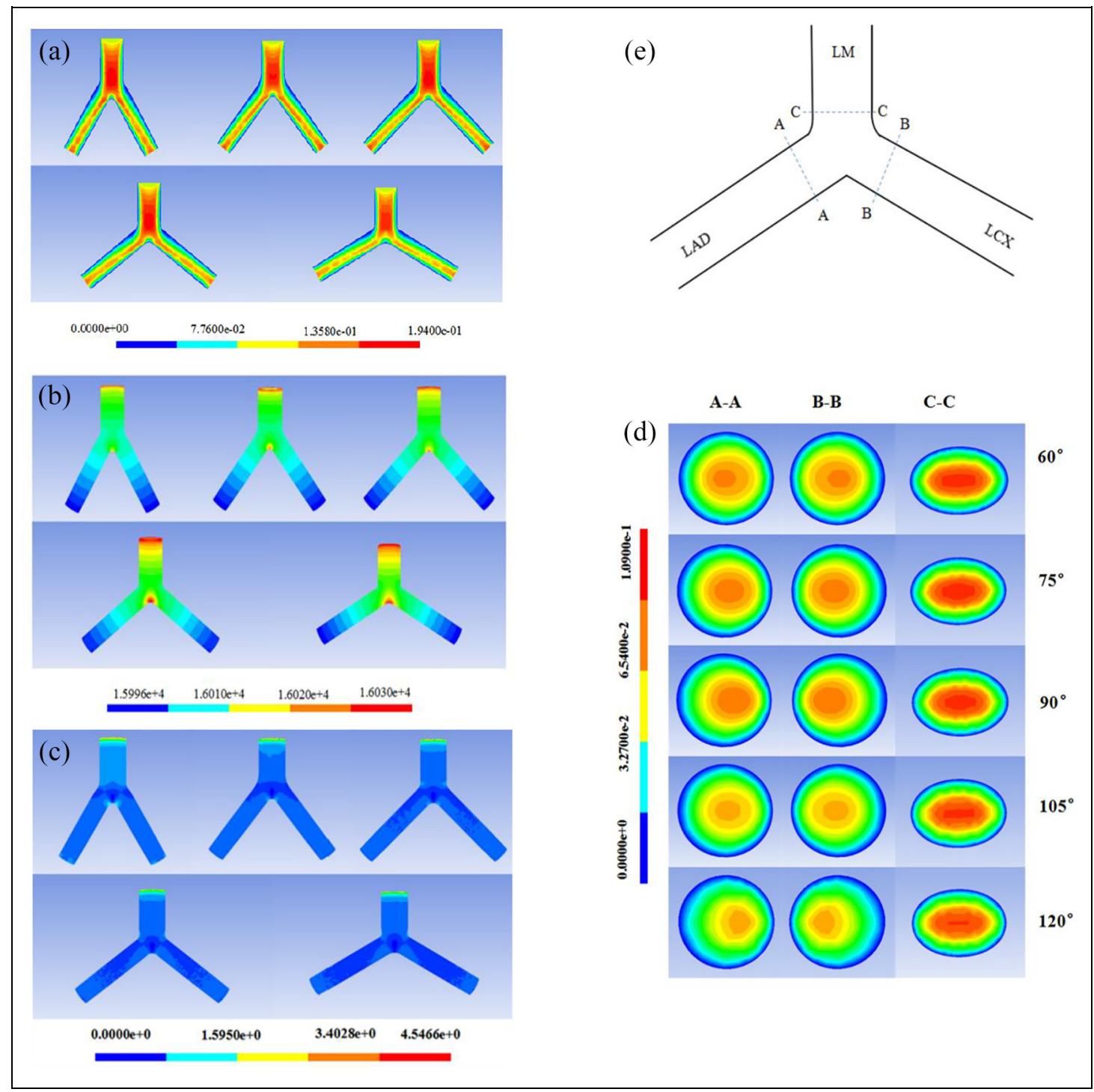

Figure 7. Contour plot of velocity in $\mathrm{m} / \mathrm{s}(\mathrm{a})$, pressure in $\mathrm{Pa}(\mathrm{b})$ and WSS distribution in $\mathrm{Pa}(\mathrm{c})$ of the left coronary idealized model with different BAs at $0.4 \mathrm{~s}$. (d) The cross-sectional velocity contour at three cross-sections (A-A, B-B and C-C) for five idealized models. (e) A-A and B-B represent the cross-section in the daughter branches; $C-C$ represents the cross-section of the main branch.

It is suggested that atherosclerosis might be formed at the time points of 0.31 and $0.5 \mathrm{~s}$; therefore, the further analysis for the formation and development of atherosclerosis at these key time points could be explored. Most recently, Pinho et al. studied the impact of the right coronary artery geometry on haemodynamic performance ${ }^{22}$ and correlation between the geometrical parameters of the LCA and haemodynamic descriptors of atherosclerosis, ${ }^{23}$ respectively. Pinho et al. found that higher angle of the right-ventricular branch leads to a haemodynamic behaviour more propitious to atherosclerosis formation. Meanwhile, they also found that higher angle between LAD and LCx was correlated with a haemodynamic behaviour propitious to plaque formation in the left LAD. These findings agree with the observation for idealized models in this study that shown the increase of BAs leading to the lower WSS in the interested areas. Through idealized CFD models, on the contrary, Chiastra et al. ${ }^{24}$ concluded that cardiac curvature has the larger effect on the local haemodynamics performance by comparing with effect of the BA. This result raised a question whether the idealized model is sufficient to provide the realistic modelling results.

\section{Limitations}

The coronary artery is one of the most complicated artery systems, with a number of the bifurcations embedded in the epicardial surface, to transport blood and nutrition to heart. In this study, the model was simplified by removing the geometrical part in the downstream, allowing the region of the first bifurcation being focused. Such simplification enables the univariate effect of angulations of bifurcation on the haemodynamic performance to be distinguished. Such 
simplification could lead to overlook the effect of the other features such as the geometrical part in the downstream on the haemodynamic performance. However, this negative effect is largely reduced when the realistic boundary conditions, the pulsatile aortic pressure and flow rate of the LCA, are applied in both inlet and outlet of the coronary arteries.

This study focused on the effect of angles between LAD and LCx on the haemodynamic performance. By comparing the simulation results from the lesionspecific model with the results from the idealized model, it was found that the flow pattern from the lesionspecific model is different from that from the idealized model even if they have the similar values of BAs. This indicates that CFD modelling of the haemodynamic mechanisms by focusing a single factor is not sufficient to explain the mechanism of the atherosclerosis formation. Therefore, a novel modelling technique, allows for the multiple parameters combining together, is required to find out the realistic haemodynamic mechanism for atherosclerosis formation and distribution.

In addition, the arterial vessel wall is assumed as rigid based on the assumption of the insignificant deformation of arterial wall occurring under the normal physiological condition. Bathe and $\mathrm{Kamm}^{25}$ concluded that assumption of the rigid arterial vessel wall has no significant effect on the real results of blood flow performance. The assumption of rigid arterial wall might not truly reflect the mechanical properties of arterial wall but simplifies the computation dramatically.

\section{Conclusion}

In this study, the idealized and patient-specific CFD models were developed to investigate the association of the angulation of bifurcation of LCA and the atherosclerosis development and distribution. Based on the simulation results and clinical observations, three areas around the region of the bifurcation have been identified as the lesion susceptible regions. It is worth to note that the lesion susceptible regions identified from both types of models were highly consistent with clinical observations which might be clinical significant to cardiovascular society. The significant correlation between BAs and the haemodynamic performance, as shown in the idealized model, was not observed from the lesionspecific modelling. This finding demonstrated that CFD modelling, which only focuses on single parameter, is not of sufficient and reliable for the clinical application. We can conclude that lesion-specific CFD modelling is an efficient and convenient way to predict the haemodynamic performance around the bifurcation region, allowing the comprehensive information for the clinicians to predict the atherosclerosis development. To assess the univariate effect on the haemodynamic performance accurately, a novel technique, which allows multiple parameters combined as a whole, is needed to provide the reliable and realistic information for clinical applications.

\section{Acknowledgements}

The authors are grateful for the material support from radiology department, Shanxi Provincial People's Hospital, China.[AQ: 6]

\section{Declaration of conflicting interests}

The author(s) declared no potential conflicts of interest with respect to the research, authorship and/or publication of this article.

\section{Funding}

The author(s) disclosed receipt of the following financial support for the research, authorship and/or publication of this article: This research is partially supported by the research grant (grant no. EP/R00 1901/1) funded by the Engineering and Physical Science and Research Council, UK.

\section{ORCID iD}

Jiling Feng (D) https://orcid.org/0000-0001-5581-0623

\section{References}

1. Chen W, Gao RL, Liu LS, et al. Cardiovascular disease report 2017 in China. Chin Circ J 2018; 33(1): 1-8.

2. Townsend N, Nichols M, Scarborough P, et al. Cardiovascular disease in Europe - epidemiological update 2015. Eur Heart J 2015; 36(40): 2696-2705.

3. Temov K and Sun Z. Coronary computed tomography angiography investigation of the association between left main coronary artery bifurcation angle and risk factors of coronary artery disease. Int $J$ Cardiovasc Imaging 2016; 32(1): 129-137.

4. White CR and Frangos JA. The shear stress of it all. Cell membrane and mechanotransduction. Philos $T R$ Soc $B$ 2007; 362(1484): 1459-1467.

5. Wentzel JJ, Whelan DM, van der Giessen WJ, et al. Coronary stent implantation changes 3-D vessel geometry and 3-D shear stress distribution. J Biomech 2000; 33(10): 1287-1295.

6. Rodriguez-Granillo GA, Rosales MA, Degrossi E, et al. Multislice CT coronary angiography for the detection of burden, morphology and distribution of atherosclerotic plaques in the left main bifurcation. Int $J$ Cardiovasc Imaging 2007; 23(3): 389-392.

7. Tang $X$ and Yuan J. The effect of coronary artery bifurcation angles on the formation of the atherosclerosis. $J$ Pract Med Imaging 2010; 11(5): 286-288.

8. Juan YH, Tsay PK, Shen WC, et al. Comparison of the left main coronary bifurcating angle among patients with normal, non-significantly and significantly stenosed left coronary arteries. Sci Rep 2017; 7: 1515.

9. Dong J, Sun Z, Inthavong K, et al. Fluid-structure interaction analysis of representative left coronary artery models with different angulations. In: Proceedings of the 
computing in cardiology conference, Zaragoza, 22-25 September 2013, pp.5-8. New York: IEEE.

10. Antoniadis AP, Giannopoulos AA, Wentzel JJ, et al. Impact of local flow haemodynamics on atherosclerosis in coronary artery bifurcations. EuroIntervention 2015;11(Suppl. V): V18-V22.

11. Beier S, Ormiston $\mathbf{J}$, Webster $\mathbf{M}$, et al. Impact of bifurcation angle and other anatomical characteristics on blood flow - a computational study of non-stented and stented coronary arteries. $J$ Biomech 2016; 49(9): 1570-1582.

12. Gnasso A, Carallo C, Irace C, et al. Association between intima-media thickness and wall shear stress in common carotid arteries in healthy male subjects. Circulation 1996; 94(12): 3257-3262.

13. Liu B, Zheng J, Bach R, et al. Influence of model boundary conditions on blood flow patterns in a patient specific stenotic right coronary artery. Biomed Eng Online 2015; 14(Suppl. 1): S6.

14. Hasan M, Rubenstein DA and Yin W. Effects of cyclic motion on coronary blood flow. J Biomech Eng 2013; 135(12): 121002.

15. Politis AK, Stavropoulos GP, Christolis MN, et al. Numerical modeling of simulated blood flow in idealized composite arterial coronary grafts: steady state simulations. J Biomech 2007; 40(5): 1125-1136.

16. Chaichana T, Sun Z and Jewkes J. Computation of hemodynamics in the left coronary artery with variable angulations. J Biomech 2011; 44(10): 1869-1878.

17. Tash OA and Razavi SE. Numerical investigation of pulsatile blood flow in a bifurcation model with a non-planar branch: the effect of different bifurcation angles and nonplanar branch. BioImpacts 2012; 2(4): 195-205.

18. Swillens A, De WM, Nordgaard H, et al. Effect of the degree of LAD stenosis on 'competitive flow' and flow field characteristics in LIMA-to-LAD bypass surgery. Med Biol Eng Comput 2012; 50(8): 839-849.

19. Brown BG, Bolson EL and Dodge HT. Dynamic mechanisms in human coronary stenosis. Circulation 1984; 70(6): 917-922.

20. Malvè M, Gharib AM, Yazdani SK, et al. Tortuosity of coronary bifurcation as a potential local risk factor for atherosclerosis: CFD steady state study based on in vivo dynamic CT measurements. Ann Biomed Eng 2015; 43(1): 82-93.

21. Chatzizisis YS, Coskun AU, Jonas M, et al. Role of endothelial shear stress in the natural history of coronary atherosclerosis and vascular remodeling: molecular, cellular, and vascular behavior. J Am Coll Cardiol 2007; 49(25): 2379-2393.

22. Pinho N, Sousa LC, Castro CF, et al. The impact of the right coronary artery geometric parameters on hemodynamic performance. Cardiovasc Eng Technol 2019; 10(2): 257-270.

23. Pinho N, Castro CF, António C, et al. Correlation between geometric parameters of the left coronary artery and hemodynamic descriptors of atherosclerosis: FSI and statistical study. Med Biol Eng Comput 2019; 57: 715729.

24. Chiastra C, Gallo D and Tasso P. Healthy and diseased coronary bifurcation geometries influence near-wall and intravascular flow: a computational exploration of the hemodynamic risk. J Biomech 2017; 58: 79-88.

25. Bathe $\mathrm{M}$ and Kamm RD. A fluid-structure interaction finite element analysis of pulsatile blood flow through a compliant stenotic artery. J Biomech Eng 1999; 121(4): 361-369. 